\title{
Análisis físico - químico e influencia de los minerales disueltos en el sabor del agua potable, de las principales plantas de tratamiento de Quito
}

\section{(Physical - chemist analysis and influence of dissolved minerals on the tap water taste from Quito's principals treatment plants)}

\author{
Stephanie Torres-Silva1, Ivan Tapia-Calvopiña², Lorena Goetschel-Gomez², Edgar Pazmiño-Salazar
}

\begin{abstract}
Resumen
El sabor del agua potable está influenciado por sustancias disueltas como: $\mathrm{Na}^{+}, \mathrm{K}^{+}, \mathrm{Fe}^{3+}, \mathrm{Cu}^{2+}, \mathrm{Ca}^{2+}, \mathrm{Mg}^{2+}, \mathrm{HCO}_{3}{ }^{-}$, $\mathrm{NO}_{3}^{-}, \mathrm{Cl}^{-}, \mathrm{SO}_{4}{ }^{2-}$ y $\mathrm{CaCO}_{3}$. El presente estudio se llevó a cabo en la Empresa Pública Metropolitana de Agua Potable y Saneamiento (Epmaps), encargada de potabilizar y distribuir el agua en Quito. Se tomaron muestras de agua en seis plantas de tratamiento durante cinco semanas y se realizaron los siguientes análisis: evaluación sensorial con un panel de jueces entrenados, que determinaron el grado de aceptación (Prueba FRA (Flavor Rating Assessment)) y el grado de satisfacción (Prueba hedónica), determinación de parámetros fisicoquímicos y de la concentración de minerales disueltos. Posteriormente, se correlacionó la concentración de minerales y el sabor, mediante análisis factorial múltiple con análisis de componentes principales. Se demostró que la concentración de minerales disueltos influye directamente en el sabor del agua potable y que, estadísticamente, el mejor método sensorial es la prueba hedónica. Todas las muestras cumplieron la norma ecuatoriana y únicamente el agua de la planta de tratamiento 2 (PT2) obtuvo menor valoración sensorial. Su sabor fue descrito como ligeramente salado, lo que se relaciona con sus concentraciones de $\mathrm{Ca}^{2+}, \mathrm{Mg}^{2+}, \mathrm{Na}^{+}, \mathrm{K}^{+}, \mathrm{HCO}_{3}{ }^{-}, \mathrm{CaCO}_{3} \mathrm{y} \mathrm{Cl}^{-}$.
\end{abstract}

\section{Palabras clave}

Agua potable; minerales disueltos; sabor; análisis sensorial, Quito.

\begin{abstract}
Drinking water flavor is influenced by some dissolved substances like $\mathrm{Na}^{+}, \mathrm{K}^{+}, \mathrm{Fe}^{3+}, \mathrm{Cu}^{2+}, \mathrm{Ca}^{2+}, \mathrm{Mg}^{2+}, \mathrm{HCO}_{3}^{-}, \mathrm{NO}_{3}^{-}, \mathrm{Cl}^{-}$, $\mathrm{SO}_{4}^{2-}$ and $\mathrm{CaCO}_{3}$. This research was conducted in the Public Metropolitan Water and Sanitation Company or EPMAPS (for its acronym in Spanish) which is in charge of water purification and distribution in Quito. It was taken samples from six different treatment plants during five weeks and the following analysis were made: Sensory analysis executed by a panel of trained judges, who evaluated the acceptance ratio (FRA Test (Flavor Rating Assessment)) and the satisfaction ratio (Hedonic Test), physiochemical parameters determination and dissolved minerals concentration. Later the minerals concentration was correlated with the water's flavor through a multiple factor analysis with main components analysis. As a result of this correlation, it was evidenced that the dissolved mineral concentration directly influences on the tap water flavor and determined statistically that the best sensory analysis is the Hedonic Test. Only the water from the treatment plant number 2 (PT2) obtained a lower sensorial valuation, whose flavor was described as lightly salted, which is related to its concentration of $\mathrm{Ca}^{+}, \mathrm{Mg}^{+}, \mathrm{Na}^{+}, \mathrm{K}^{+}, \mathrm{HCO}_{3}^{-}, \mathrm{CaCO}_{3}$ y $\mathrm{Cl}^{-}$.
\end{abstract}

\section{Keywords}

Drinking water; dissolved minerals; taste; sensory analysis, Quito. 


\section{Introducción}

El agua que es consumida por el ser humano, debe cumplir con parámetros de calidad, inocuidad y con expectativas en cuanto al sabor (Rahman I., 2017). La detección de un determinado sabor u olor anormal en el agua está asociada a una percepción de baja calidad (Sancho, 2002), por lo que es necesaria su evaluación sensorial. Los consumidores son muy sensibles respecto al agua para beber, por eso los proveedores deben mantener su calidad en los niveles más altos posibles. Las pruebas, diseños y análisis deben abordarse de manera que permitan optimizar la sensibilidad, validez y confiabilidad (Burlingame G., 2017). Por otro lado, el desarrollo de un protocolo simple, para formar un panel sensorial conformado por un mínimo de 6 jueces especialmente seleccionados y capacitados, es una herramienta útil para evaluar la calidad de un alimento o bebida (Salguero, 2013), y el uso de herramientas estadísticas multivariables se aplica en la evaluación sensorial (Villarroel, 2003).

El contenido inorgánico del agua potable se ve afectado por el tratamiento químico y por la composición hidrogeoquímica de las aguas subterráneas o de superficie, incluyendo ríos y embalses (Dinelli E., 2012); además, la naturaleza y la concentración de minerales disueltos afectan su sabor y aceptación (Vingerhoeds, 2017; Wang, 2016). Las sustancias químicas presentes en el agua potable dependen de varios factores, como: la fuente de agua (con relación al contenido de $\mathrm{Fe}, \mathrm{Cu}, \mathrm{Zn}, \mathrm{Al}, \mathrm{Ca}, \mathrm{Mg}$, carbonatos, bicarbonatos, sulfatos, cloruros y contaminantes como la geosmina), el método de tratamiento (cloro, cloraminas, ozono) y los sistemas de distribución (Fe, Cu, y polímeros como hidrocarburos). Adicionalmente, el agua puede influir significativamente en el sabor de alimentos y bebidas (Vingerhoeds, 2017), y su composición contribuye a la ingesta diaria de minerales requeridos por el ser humano desde el punto de vista nutricional (Azoulay A., 2001); (Perry, 2013).

En el estudio "Influencia de minerales en el sabor del agua embotellada y el agua de grifo, un enfoque quimiométrico" (Platikanov S., 2013), que se realizó en España, se evaluó el grado de aceptación de varias muestras de agua, mediante pruebas sensoriales con jueces entrenados y se comparó trece propiedades fisicoquímicas mediante ACP (Análisis de Componentes Principales). Los resultados mostraron que, las muestras de las dos clases de agua que tuvieron más aceptación, se asociaron a un contenido medio de sólidos totales disueltos, a la alta concentración de $\mathrm{HCO}_{3}$; $\mathrm{SO}_{4}^{2-}, \mathrm{Ca}^{2+}$ y $\mathrm{Mn}^{2+}$, y a valores de $\mathrm{pH}$ relativamente altos en el agua potable. Las altas concentraciones de $\mathrm{Na}^{+}, \mathrm{K}^{+}$y $\mathrm{Cl}^{-}$fueron percibidas por varios jueces, mientras que el cloro residual no tuvo ningún efecto en los resultados, y los jueces no pudieron distinguir entre agua embotellada y agua potable.

En otro estudio se investigó la preferencia de tres tipos de agua: agua embotellada, agua potable de grifo y agua potable reciclada, mediante una prueba de sabor a ciegas realizada por 143 participantes. Los evaluadores prefirieron el agua embotellada y el agua reciclada, las que fueron tratadas con ósmosis inversa, sobre el agua potable de grifo que presentó niveles de pH más altos y concentraciones más bajas de Ca y $\mathrm{HCO}_{3}{ }^{-}$(Harmon D., 2018).

En una investigación sobre la influencia de diferentes concentraciones de hierro y dureza del agua, en la percepción del sabor de bebidas endulzadas con diferentes edulcorantes mediante una prueba hedónica de 9 puntos, el Fe ${ }^{2+}$ creó un sabor metálico en el agua y disgustó a los consumidores, mientras que $\mathrm{Ca}^{2+}, \mathrm{Mg}^{2+}$ y Na${ }^{+}$casi no tuvieron impacto en la percepción del sabor. Además, la interacción entre la sacarosa y los iones ferrosos aumentó significativamente la aceptación del agua muy dura, y la percepción del sabor dulce (Wang A., 2016) . 
El papel de los sulfatos y nitratos en el sabor del agua potable depende del umbral de concentración, lo que se comprobó mediante la realización de pruebas dúo-trio con un panel entrenado, que permitieron determinar que el umbral de detección de $\mathrm{NO}_{3}$ - es alto (100 mg/l), y que no es relevante para el sabor del agua. Por el contrario, el $75 \%$ de los jueces percibieron como salado y amargo el sabor del agua con una concentración de $160 \mathrm{mg} / \mathrm{l} \mathrm{de} \mathrm{Na}_{2}\left(\mathrm{SO}_{4}\right)$ y de $80 \mathrm{mg} / \mathrm{l}$ de $\mathrm{CaSO}_{4}$ (López et al., 2017).

La Usepa (United States Environmental Protection Agency), estableció 15 parámetros del agua potable que producen efectos organolépticos (Al, Cl, color, Cu, corrosividad, F, agentes espumantes, Fe, Mn, olor, pH, Ag, SO ${ }_{4}^{2-}$, sólidos disueltos totales y Zn) bajo la ley 40 CFR 141. 208 (Usepa, 2020), información que sirvió de base para el estudio de Dietrich y Burlingame en el 2020 (p. 1), quienes asociaron la presencia de 21 sustancias químicas puras a concentraciones mayores al umbral del olor y sabor, mediante el uso de descriptores como "salado" para altas concentraciones de sodio, y "clorado" para el cloro libre. Estos descriptores ayudan a evitar confusión en los consumidores y llegar a un consenso sobre el sabor. Los mismos autores, en una publicación anterior, indican que las concentraciones altas de $\mathrm{Cl}, \mathrm{Cu}, \mathrm{F}, \mathrm{Fe}$ y Mn tienen efectos organolépticos, lo que afecta la calidad percibida por los consumidores del agua potable (Dietrich y Burlingame, 2014).

Los minerales presentes en el agua, además de influir en su calidad, al ser ingeridos por el consumidor aportan a la ingesta diaria recomendada de estos micronutrientes, según se explica en el estudio "El contenido mineral en el agua de grifo en los hogares de Estados Unidos" (Perry, 2013), donde se analizó el contenido de ocho minerales: Na, K, Ca, Mg, Fe, Cu, Mn, P y Zn, en muestras del agua de grifo residencial de este país, que sirvió para generar los datos actuales de la Base de Datos Nacional de Nutrientes de la USDA. Suponiendo un consumo diario de un litro de agua del grifo por persona, se concluyó que proporciona en promedio más del 1 \% de la ingesta diaria recomendada de cuatro minerales ( $\mathrm{Cu}, \mathrm{Ca}, \mathrm{Mg}$ y Na), de los ocho estudiados.

En el campo industrial se observó el efecto de la calidad del agua en el sabor del té verde y el té negro, al preparar los dos tipos de té con diferentes clases de agua. Se analizó el color, la turbidez, el contenido de epicatequinas y el sabor de las dos clases de infusiones y se concluyó que el tipo de agua afecta la aceptabilidad por parte del consumidor, ya que el agua desionizada o purificada extrae del té mayor contenido de catequinas que aportan al sabor de la infusión (Franks, 2019).

En el Ecuador no existen datos sobre la correlación existente entre el sabor y la presencia de minerales en el agua potable, a pesar de que las estadísticas indican que, en ciudades como Quito, cada habitante consume un valor estimado de 200 litros de agua potable al mes, de los que ingiere como bebida aproximadamente 2 litros al día (INEC, 2013), lo que justifica la presente investigación.

El objetivo principal de este estudio fue determinar la influencia de minerales disueltos $\mathrm{Na}^{+}$, $\left.\mathrm{K}^{+}, \mathrm{Fe}^{3+}, \mathrm{Cu}^{2+}, \mathrm{Ca}^{2+}, \mathrm{Mg}^{2+}, \mathrm{CaCO}_{3}, \mathrm{HCO}_{3}-\mathrm{NO}_{3}-\mathrm{Cl}^{-}, \mathrm{SO}_{4}{ }^{2-}\right)$ en el sabor del agua, mediante análisis físicoquímico y sensorial de las muestras de agua potable obtenidas en seis plantas de tratamiento de la ciudad de Quito, a fin de comprobar la hipótesis formulada que indica, que el contenido de minerales disueltos influye en el grado de aceptación y satisfacción sensorial del agua potable.

\section{Metodología}

El estudio se llevó a cabo en las plantas de tratamiento y en el laboratorio de la Empresa Pública Metropolitana de Agua Potable y Saneamiento (Epmaps), en cuyas instalaciones se recibe, trata y potabiliza el agua destinada al Distrito Metropolitano de Quito. 
2.1. Muestreo: Para tomar una muestra representativa de cada planta, se realizó un plan de muestreo que contempló tomar cinco muestras (una cada semana), de las seis plantas de tratamiento que generan mayor volumen de agua potable de la Epmaps (30 muestras en total), antes de su distribución y abastecimiento hacia la mayoría de los barrios de Quito DM, durante los meses comprendidos entre mayo y julio del 2017. Se analizaron seis parámetros físico-químicos generales de cada muestra (180 ensayos) y once minerales por cinco repeticiones (total: 1650 datos), y dos análisis sensoriales (prueba FRA y prueba hedónica) de cada muestra semanal (60 análisis sensoriales) con un panel de cata que tuvo mínimo seis jueces entrenados para cada semana, según lo descrito en la bibliografía (Salguero, 2013). En todas las plantas se analizó las muestras antes y después del tratamiento de potabilización.

2.2. Análisis físico-químicos: El contenido de cloro libre residual se analizó con un medidor de cloro digital DPD, marca LaMotte 1200, mediante reacción colorimétrica in situ (Standard Methods 4500-Cl-G, 2012). El pH se midió con un potenciómetro, marca InoLab nivel 1 modelo ba12217e (Standard Methods 4500-H+-B, 2012); la conductividad y sólidos totales disueltos por conductimetría (Standard Methods 2510-B, 2012) en un conductímetro marca InoLab nivel 1 multi parámetros modelo 1F10-220, color por comparación visual, utilizando un colorímetro marca Agua Tester Modelo 611-A (Standard Methods 2120-B, 2012); y la turbiedad por el método nefelométrico (Standard Methods 2130-B, 2012).

2.3. Análisis de minerales disueltos: El contenido de sodio y potasio se determinó en un espectrofotómetro de absorción atómica Varian Modelo AA240FS, a una longitud de onda de $589.0 \mathrm{~nm}$ y $766.5 \mathrm{~nm}$ respectivamente, mientras que la concentración de hierro y cobre se obtuvo por absorción atómica con lámpara multielementos (Co/Cr/Cu/Fe/Mn/Ni), mediante aspiración directa en llama de aire - acetileno, en el mismo equipo en el que se analizó el sodio y potasio, a una longitud de onda de $324.8 \mathrm{~nm}$ para el cobre, y $248.3 \mathrm{~nm}$ para el hierro (Standard Methods 3111-B, 2012).

La cuantificación de calcio se realizó por medio de la determinación de la dureza cálcica por titulación con EDTA (Standard Methods 3500-Ca-B, 2012), mediante el uso de una bureta digital, y la de magnesio por cálculo, utilizando este mismo método para determinar dureza total (NTE INEN 974, 2016) (Standard Methods 3500-Mg-B, 2012).

La concentración de carbonato de calcio se determinó a través de la medición de la alcalinidad por titulación con ácido sulfúrico (INEN ISO 9963-1, 2014), el contenido de bicarbonato de calcio se obtuvo por cálculo, y la de cloruros por titulación con nitrato de plata (Standard Methods 4500-Cl-B, 2012). Para las determinaciones analíticas se utilizaron buretas digitales.

La cantidad de nitratos se analizó por screening ultravioleta a una longitud de onda de 275 nm (Standard Methods 4500-NO3-B, 2012) y la de sulfatos por espectrofotometría a una longitud de onda de $420 \mathrm{~nm}$ (Standard Methods 4500-SO4-E, 2012) en el espectrofotómetro UV - VIS marca Varian modelo CARY50.

2.4. Análisis sensorial: La evaluación del sabor del agua se realizó mediante la aplicación de dos pruebas sensoriales. Inicialmente se efectuó la prueba FRA (Flavor Rating Assessment), modificada de la Epmaps, que utiliza una escala de 5 puntos, donde 1=Estaría encantado de aceptar esta agua como mi agua de consumo diario y $5=$ No podría aceptar esta agua como mi agua de consumo diario (Standard Methods 2160-C, 2012). Luego de un receso de 10 minutos, los jueces realizaron la prueba hedónica de 9 puntos, cuya escala se puntúa de 1 a 9, donde: 1=Extremadamente agradable y $9=$ Extremadamente desagradable (Sancho, 2002). Se utilizó un diseño de bloques completamente al azar (DBCA), Anova de dos factores, con una muestra 
por grupo, donde el factor A son las muestras de agua potable de cada planta de tratamiento y el factor B es la variabilidad de los resultados de los jueces entrenados, además de analizar el contraste entre promedios basado en la diferencia mínima significativa (DMS).

2.5. Análisis estadístico: Se procesaron los 1650 datos, obtenidos de la concentración de minerales disueltos $\left(\mathrm{Na}^{+}, \mathrm{K}^{+}, \mathrm{Fe}^{3+}, \mathrm{Cu}^{2+}, \mathrm{Ca}^{2+}, \mathrm{Mg}^{2+}, \mathrm{CaCO}_{3}, \mathrm{HCO}_{3}-\mathrm{NO}_{3}, \mathrm{Cl}^{-}, \mathrm{SO}_{4}{ }^{2-}\right)$, mediante un diseño anidado o jerárquico con la ayuda del software estadístico Statgraphics Centurion versión 17, donde el factor A corresponde a las muestras de agua potable de cada planta de tratamiento y el factor B a la semana de muestreo. Se utilizó el diseño anidado porque existe una jerarquía o anidamiento de los niveles del segundo factor en cada uno de los niveles del primero. Además, este diseño permitió evaluar la concentración de once analitos que pertenecen a seis plantas de tratamiento (A). De cada planta de tratamiento se realizó el análisis durante cinco semanas de muestreo (B), sobre las cuales se realizan cinco repeticiones (C). Se observó mediante este diseño que la mayor variación proviene de las plantas de tratamiento (A), seguido de la semana de muestreo (B) y que existe muy poca contribución a la variabilidad otorga por el factor de repeticiones (C).

Finalmente, para correlacionar el sabor del agua con la concentración de los minerales estudiados, se efectuó el análisis estadístico multifactorial y el análisis de componentes principales (ACP) con la ayuda del software estadístico Minitab versión 17.

\section{Resultados y discusión}

\subsection{Análisis físico-químicos}

Los parámetros generales de calidad del agua que se analizaron, son: cloro libre residual, pH, conductividad, sólidos totales disueltos (STD), color y turbiedad. Los resultados se muestran en la tabla 1, en la que se puede apreciar que existe variación de los valores en las cinco semanas de muestreo en todas las plantas. Se determinó que PT2 tuvo mayor conductividad y mayor contenido de STD, lo que se relaciona con la palatabilidad, ya que una concentración de STD menor a $600 \mathrm{mg} / \mathrm{l}$ corresponde a agua de buena palatabilidad, mientras que, a valores mayores de conductividad, la palatabilidad disminuye significativa y progresivamente (OMS, 2006). Además, la conductividad y STD se relacionan directamente, por lo que, la conductividad influye de manera inversa en el sabor del agua, según se describe posteriormente.

Los resultados coinciden con lo señalado en 2017 por Virgerhoeds, quien indica que el parámetro STD es un determinante importante en la percepción del agua (pp. 42-57), y que el agua tiene sabor más fresco a una concentración de STD entre 19 y 350 mg/l. Otro aspecto a considerar en esta publicación es que la calidad sensorial percibida del agua mejora al realizar un proceso de ósmosis inversa y de remineralización.

En cuanto a los demás parámetros generales, todas las muestras cumplieron con los niveles indicados en la Norma Técnica Ecuatoriana del Agua Potable (INEN 1108, 2020), que son: turbiedad menor a $5 \mathrm{NTU}$, sabor y olor aceptables, cloro libre residual de 0.3 a $1.5 \mathrm{mg} / \mathrm{l}$, y color aparente máximo de 15 UCA (unidades de color aparente). 
Tabla 1. Valores promedios de parámetros generales de calidad de agua durante los meses de mayo a julio del 2017 en Epmaps.

\begin{tabular}{|c|c|c|c|c|c|c|c|}
\hline \multirow{2}{*}{ Parámetro general } & \multicolumn{7}{|c|}{ PLANTAS DE TRATAMIENTO } \\
\cline { 2 - 8 } & $\mathrm{n}$ & PT1 & PT2 & PT3 & PT4 & PT5 & PT6 \\
\hline Cloro libre residual $(\mathrm{mg} / \mathrm{l})$ & 30 & $1.09 \pm 0.16$ & $1.13 \pm 0.67$ & $1.18 \pm 0.13$ & $1.25 \pm 0.16$ & $1.20 \pm 0.16$ & $1.15 \pm 0.16$ \\
\hline $\mathrm{pH}$ & 30 & $7.34 \pm 0.21$ & $7.29 \pm 0.07$ & $7.08 \pm 0.15$ & $7.24 \pm 0.17$ & $7.31 \pm 0.43$ & $7.33 \pm 0.24$ \\
\hline Conductividad $(\mu \mathrm{S} / \mathrm{cm})$ & 30 & $104.9 \pm 19.6$ & $726.4 \pm 3.1$ & $208.3 \pm 21.9$ & $212.2 \pm 49.9$ & $172.2 \pm 63.8$ & $99.8 \pm 21.1$ \\
\hline STD (mg/l) & 30 & $53 \pm 10$ & $363 \pm 2$ & $104 \pm 11$ & $106 \pm 25$ & $86 \pm 32$ & $50 \pm 11$ \\
\hline Color (UCA Pt-Co) & 30 & $0 \pm 0$ & $0 \pm 0$ & $1 \pm 3$ & $2 \pm 3$ & $1 \pm 7$ & $0 \pm 0$ \\
\hline Turbiedad (NTU) & 30 & $0.509 \pm 0.014$ & $0.500 \pm 0.161$ & $0.500 \pm 0.000$ & $0.693 \pm 0.201$ & $0.596 \pm 1.726$ & $0.536 \pm 0.000$ \\
\hline
\end{tabular}

n= número de ensayos realizados.

Como resumen de los resultados, la tabla 2 indica los promedios y desviaciones estándar de todos los minerales analizados en las plantas de tratamiento.

Tabla 2. Concentración de minerales promedios en agua potable durante los meses de mayo a julio del 2017 en Epmaps.

\begin{tabular}{|c|c|c|c|c|c|c|c|c|c|c|c|c|c|c|c|c|c|c|c|}
\hline \multirow{3}{*}{$\begin{array}{c}\begin{array}{c}\text { Mine- } \\
\text { rales } \\
(\mathrm{mg} / \mathrm{l})\end{array} \\
\mathrm{Na}^{+}\end{array}$} & \multicolumn{19}{|c|}{ PLANTAS DE TRATAMIENTO } \\
\hline & \multirow{2}{*}{\begin{tabular}{|c|}
$\mathbf{n}$ \\
150
\end{tabular}} & \multicolumn{3}{|c|}{ PT1 } & \multicolumn{3}{|c|}{ РT2 } & \multicolumn{3}{|c|}{ РT3 } & \multicolumn{3}{|c|}{ PT4 } & \multicolumn{3}{|c|}{ PT5 } & \multicolumn{3}{|c|}{ PT6 } \\
\hline & & 6.70 & \pm & 2.50 & 59.37 & \pm & 18.83 & 13.72 & \pm & 4,45 & 12.02 & \pm & 2.78 & 10.42 & \pm & 5.06 & 8.04 & \pm & 3.90 \\
\hline $\mathrm{K}^{+}$ & 150 & 1.31 & \pm & 0.31 & 6.51 & \pm & 1.57 & 3.58 & \pm & 1,15 & 2.80 & \pm & 0.54 & 2.90 & \pm & 0.53 & 1.32 & \pm & 0.11 \\
\hline $\mathrm{Ca}^{2+}$ & 150 & 7.58 & \pm & 1.79 & 20.62 & \pm & 2.84 & 13.16 & \pm & 1,87 & 16.79 & \pm & 1.75 & 11.37 & \pm & 4.80 & 7.70 & \pm & 1.81 \\
\hline $\mathrm{Mg}^{2+}$ & 150 & 3.72 & \pm & 1.19 & 41.63 & \pm & 12.85 & 8.35 & \pm & 1,47 & 8.03 & \pm & 0.38 & 7.11 & \pm & 3.76 & 3.35 & \pm & 0.47 \\
\hline $\mathrm{HCO}_{3}^{-}$ & 150 & 30.10 & \pm & 6.40 & 344.73 & \pm & 86.10 & 65.98 & \pm & 16,53 & 84.53 & \pm & 6.15 & 65.45 & \pm & 40.39 & 29.25 & \pm & 6.43 \\
\hline $\mathrm{CaCO}_{3}$ & 150 & 26.74 & \pm & 6.65 & 270.19 & \pm & 70.33 & 55.78 & \pm & 15,48 & 69.62 & \pm & 5.53 & 54.38 & \pm & 34.82 & 25.59 & \pm & 7.90 \\
\hline $\mathrm{SO}_{4}^{-}$ & 150 & 15.83 & \pm & 4.45 & 27.04 & \pm & 13.68 & 39.12 & \pm & 1,46 & 28.89 & \pm & 3.20 & 27.24 & \pm & 9.65 & 13.42 & \pm & 2.79 \\
\hline $\mathrm{Cl}^{-}$ & 150 & 8.82 & \pm & 2.77 & 36.17 & \pm & 14.08 & 9.13 & \pm & 1,99 & 9.01 & \pm & 3.05 & 7.77 & \pm & 3.46 & 8.35 & \pm & 3.34 \\
\hline $\mathrm{Fe}$ & 150 & \multicolumn{3}{|c|}{$<0.05$} & \multicolumn{3}{|c|}{$<0.05$} & \multicolumn{3}{|c|}{$<0.05$} & \multicolumn{3}{|c|}{$<0.05$} & \multicolumn{3}{|c|}{$<0.05$} & \multicolumn{3}{|c|}{$<0.05$} \\
\hline $\mathrm{Cu}$ & 150 & \multicolumn{3}{|c|}{$<0.014$} & \multicolumn{3}{|c|}{$<0.014$} & \multicolumn{3}{|c|}{$<0.014$} & \multicolumn{3}{|c|}{$<0.014$} & \multicolumn{3}{|c|}{$<0.014$} & \multicolumn{3}{|c|}{$<0.014$} \\
\hline $\mathrm{NO}_{3}$ & 150 & \multicolumn{3}{|c|}{$<0.001$} & 3.79 & \pm & 0.42 & \multicolumn{3}{|c|}{$<0.001$} & \multicolumn{3}{|c|}{$<0.001$} & \multicolumn{3}{|c|}{$<0.001$} & \multicolumn{3}{|c|}{$<0.001$} \\
\hline
\end{tabular}

n= número de ensayos realizados.

A partir de los datos de la tabla 2, se realizó el análisis estadístico de diseño anidado o jerárquico (tabla 3), que permite explicar la influencia de la cantidad de minerales, mediante la estimación de varianza de estos parámetros, en dos fuentes estadísticas que son plantas de tratamiento y semana de muestreo del agua potable. El resultado indica que estadísticamente el porcentaje de varianza más alto y cercano al $100 \%$ corresponde a la concentración de $\mathrm{Na}^{+}, \mathrm{K}^{+}, \mathrm{Ca}^{2+}, \mathrm{Mg}^{2+}$ $\mathrm{HCO}_{3}$, y $\mathrm{CaCO}_{3^{\prime}}$ que varía significativamente con relación a la fuente: plantas de tratamiento. 
No se realizó el análisis de varianza mediante el diseño anidado para el contenido de $\mathrm{Fe}^{3+}$, $\mathrm{Cu}^{2+}, \mathrm{y} \mathrm{NO}_{3}$, debido a que los datos fueron menores al límite de cuantificación. Estos resultados contrastan con los obtenidos en 2013 por Platikanov et al., quienes demostraron que estos tres parámetros sí influyen en el sabor del agua. Cabe indicar que todas las muestras cumplieron con los requisitos de la NTE (INEN 1108, 2020) en lo referente al límite máximo permitido de $\mathrm{Cu}^{2+}$ que es $2,0 \mathrm{mg} / \mathrm{l}$ y de $\mathrm{NO}_{3}$ - que es $50 \mathrm{mg} / \mathrm{l}$. El contenido máximo de hierro no se especifica en la norma.

Tabla 3. Diseño anidado o jerárquico de dos factores.

Respuesta experimental: concentración en (mg/l) de minerales

\begin{tabular}{|c|c|c|c|c|}
\hline Mineral & Fuente & $\mathrm{F}$ & Valor crítico & $\% S^{2}$ \\
\hline \multirow{2}{*}{$\mathrm{Na}^{+}$} & Planta de tratamiento (A) & 5917.970 & 2.620 & 89.66 \\
\hline & Semana de muestreo (B) & 53.080 & 1.610 & 9.95 \\
\hline \multirow{2}{*}{$\mathrm{K}^{+}$} & Planta de tratamiento (A) & 377521.550 & 2.620 & 84.22 \\
\hline & Semana de muestreo (B) & 5572.860 & 1.610 & 15.77 \\
\hline \multirow{2}{*}{$\mathrm{Ca}^{2+}$} & Planta de tratamiento (A) & 3640.470 & 2.620 & 66.17 \\
\hline & Semana de muestreo (B) & 142.060 & 1.610 & 33.35 \\
\hline \multirow{2}{*}{$\mathrm{Mg}^{2+}$} & Planta de tratamiento (A) & 4841.890 & 2.620 & 96.56 \\
\hline & Semana de muestreo (B) & 12.760 & 1.610 & 2.94 \\
\hline \multirow{2}{*}{$\mathrm{HCO}_{3}^{-}$} & Planta de tratamiento (A) & 9489.760 & 2.620 & 95.80 \\
\hline & Semana de muestreo (B) & 32.210 & 1.610 & 3.95 \\
\hline \multirow{2}{*}{$\mathrm{CaCO}_{3}$} & Planta de tratamiento (A) & 319917.220 & 2.620 & 90.52 \\
\hline & Semana de muestreo (B) & 2656.760 & 1.610 & 9.47 \\
\hline \multirow{2}{*}{$\mathrm{SO}_{4}^{2-}$} & Planta de tratamiento (A) & 21.320 & 2.620 & 38.50 \\
\hline & Semana de muestreo (B) & 1.530 & 1.610 & 12.80 \\
\hline \multirow{2}{*}{$\mathrm{Cl}^{-}$} & Planta de tratamiento $(\mathrm{A})$ & 23.150 & 2.620 & 33.50 \\
\hline & Semana de muestreo (B) & 2.310 & 1.610 & 26.30 \\
\hline
\end{tabular}

Según se detalla en la tabla 4, el contenido de minerales en el agua cruda presenta variaciones en las diferentes plantas y luego del tratamiento los valores de cada parámetro fisicoquímico disminuyen o se incrementan. Por ejemplo, los sulfatos y el cloro libre residual, que son residuos de agentes químicos agregados en la potabilización, no influyen en el sabor y se incrementan cuando se potabiliza del agua. En tanto que los demás parámetros disminuyen, como es el caso del hierro, cuyos valores fueron menores al límite de detección en todas las muestras, al igual que el contenido de $\mathrm{NO}_{3}$ - para PT2, PT1, PT4 y PT6. La variación de los demás parámetros fue menor y se puede observar que PT2 presenta mayor concentración de todos los analitos, tanto en el agua cruda como en la tratada. 
Tabla 4. Comparación de agua cruda AC (antes) con agua tratada AT (después), durante los meses de mayo a julio del 2017

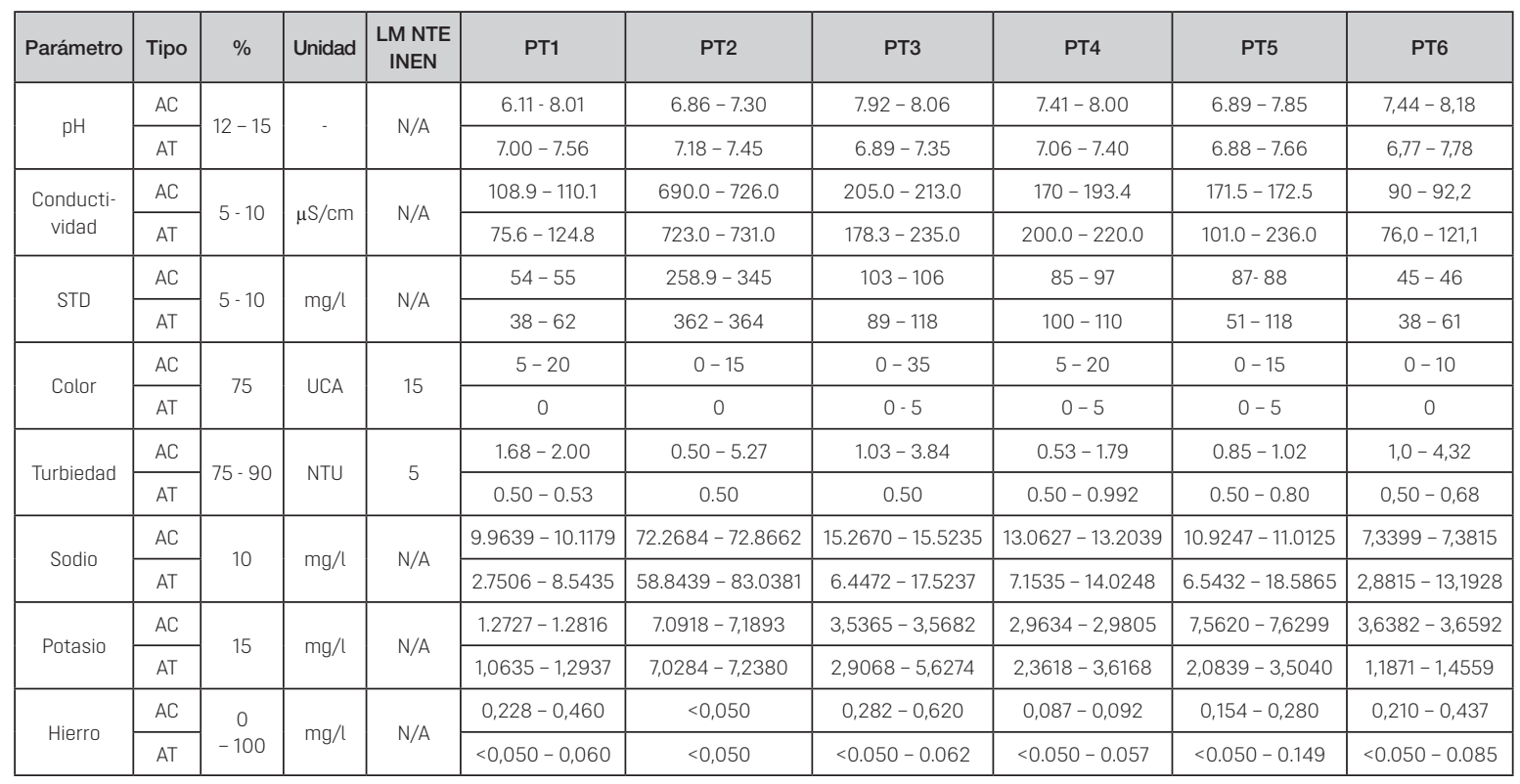

\%: porcentaje aproximado de reducción o aumento en promedio. LM: Límite máximo permitido

Continuación Tabla 4

\begin{tabular}{|c|c|c|c|c|c|c|c|c|c|c|}
\hline Parámetro & Tipo & $\%$ & Unidad & $\begin{array}{l}\text { LM NTE } \\
\text { INEN }\end{array}$ & PT1 & PT2 & PT3 & PT4 & PT5 & PT6 \\
\hline Cobre & $A C$ & 0 & mg/l & 2.0 & $<0.014$ & $<0.014$ & $<0.014$ & $<0.014$ & $<0.014$ & $<0.014$ \\
\hline \multirow{2}{*}{ Calcio } & $A C$ & \multirow{2}{*}{10} & \multirow{2}{*}{ mg/l } & \multirow{2}{*}{$\mathrm{N} / \mathrm{A}$} & $7.34-8.16$ & $26.76-27.74$ & $11.26-12.73$ & $10.93-11.42$ & $8.81-9.96$ & $4.24-4.90$ \\
\hline & AT & & & & $4.45-8.69$ & $19.55-23.57$ & $10.74-15.21$ & $16.89-18.33$ & $5.88-16.18$ & $5.30-10.22$ \\
\hline \multirow{2}{*}{$\begin{array}{c}\text { Bicarbonato } \\
\text { de calcio }\end{array}$} & $A C$ & \multirow{2}{*}{20} & \multirow{2}{*}{ mg/l } & \multirow{2}{*}{ N/A } & $43.33-45.14$ & $377.82-380.53$ & $97.50-102.02$ & $95.70-112.85$ & $85.31-94.79$ & $44.69-58.68$ \\
\hline & AT & & & & $20.90-37.11$ & $375.20-391.69$ & $42.52-87.30$ & $74.57-91.32$ & $23.47-127.25$ & $20.90-37.92$ \\
\hline \multirow{2}{*}{$\begin{array}{c}\text { Carbonato } \\
\text { de calcio }\end{array}$} & $A C$ & \multirow{2}{*}{30} & \multirow{2}{*}{ mg/l } & \multirow{2}{*}{$\mathrm{N} / \mathrm{A}$} & $35.52-37.00$ & $309.69-311.91$ & $79.92-83.62$ & $78.44-92.50$ & $69.93-77.70$ & $36.63-48.10$ \\
\hline & AT & & & & $17.13-34.10$ & $246.57-321.06$ & $38.55-72.40$ & $61.12-76.52$ & $19.24-104.30$ & $17.13-36.38$ \\
\hline \multirow{2}{*}{ Nitratos } & $\mathrm{AC}$ & \multirow{2}{*}{$\begin{array}{c}0 \\
-100\end{array}$} & \multirow{2}{*}{ mg/l } & \multirow{2}{*}{50} & $<2.215$ & $2.981-3.438$ & $0.443-0.549$ & $<2.215$ & $0.665-0.793$ & $<2.215$ \\
\hline & AT & & & & $<2.215$ & $0.000-3.743$ & $<2.215$ & $<2.215$ & $<2.215$ & $<2.215$ \\
\hline \multirow{2}{*}{ Cloruros } & $A C$ & \multirow{2}{*}{$\begin{array}{c}0 \\
-100\end{array}$} & \multirow{2}{*}{ mg/l } & \multirow{2}{*}{ N/A } & $1.94-3.89$ & $6.24-41.77$ & $1.94-3.89$ & $0.97-1.94$ & $0.97-1.94$ & $0.97-3.89$ \\
\hline & AT & & & & $6.4-12.5$ & $37.7-45.1$ & $7.4-12.3$ & $4.5-12.6$ & $2.5-11.3$ & $6.2-12.3$ \\
\hline
\end{tabular}

\%: porcentaje aproximado de reducción o aumento en promedio. LM: Límite máximo permitido.

\subsection{Análisis sensorial y correlación con el contenido de minerales}

En la figura 1 se observa la relación existente entre las muestras de las diferentes plantas de tratamiento y la apreciación numérica de los jueces en las dos pruebas sensoriales. Al aplicar 
la prueba FRA se determinó que PT2 (Planta de tratamiento 2) se acerca a un valor aproximado de: $3=$ Tal vez podría aceptar esta agua como mi agua de consumo diario, mientras que las otras plantas a: $2=$ =Estoy seguro de que podría aceptar esta agua de consumo diario. De igual manera, en la prueba hedónica PT2 se acerca a: 5=Ni agradable ni desagradable, PT1 y PT5 a: 3=Agradable, y PT3, PT4 y PT6 a: 4=Ligeramente agradable.

Al realizar el Anova de 2 factores DBCA, se determinó que en la semana de muestreo 1, el factor plantas de tratamiento es significativo (representado con un *), para la prueba FRA y el mismo factor es significativo en las semanas de muestreo 2, 3, 4 y 5 para la prueba hedónica, como se muestra en la tabla 5. Además, con la DMS se mostró que el promedio de la muestra PT2 mantuvo una diferencia significativa con respecto a las demás muestras. Estadísticamente la prueba FRA de grado de aceptación no permitió diferenciar entre muestras, mientras que la prueba hedónica permitió realizar esta diferenciación y posteriormente correlacionar con los parámetros físico-químicos.

Figura 1. Apreciación de los jueces entrenados vs plantas de tratamiento del agua potable evaluada

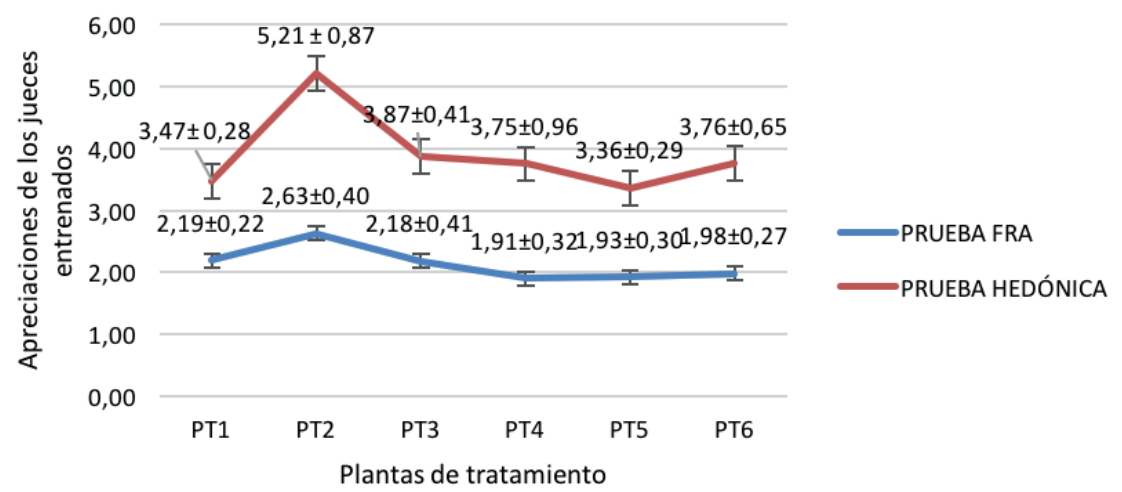

Tabla 5. Análisis de varianza y comparación de las medias de los resultados entre las plantas de tratamiento por DMS

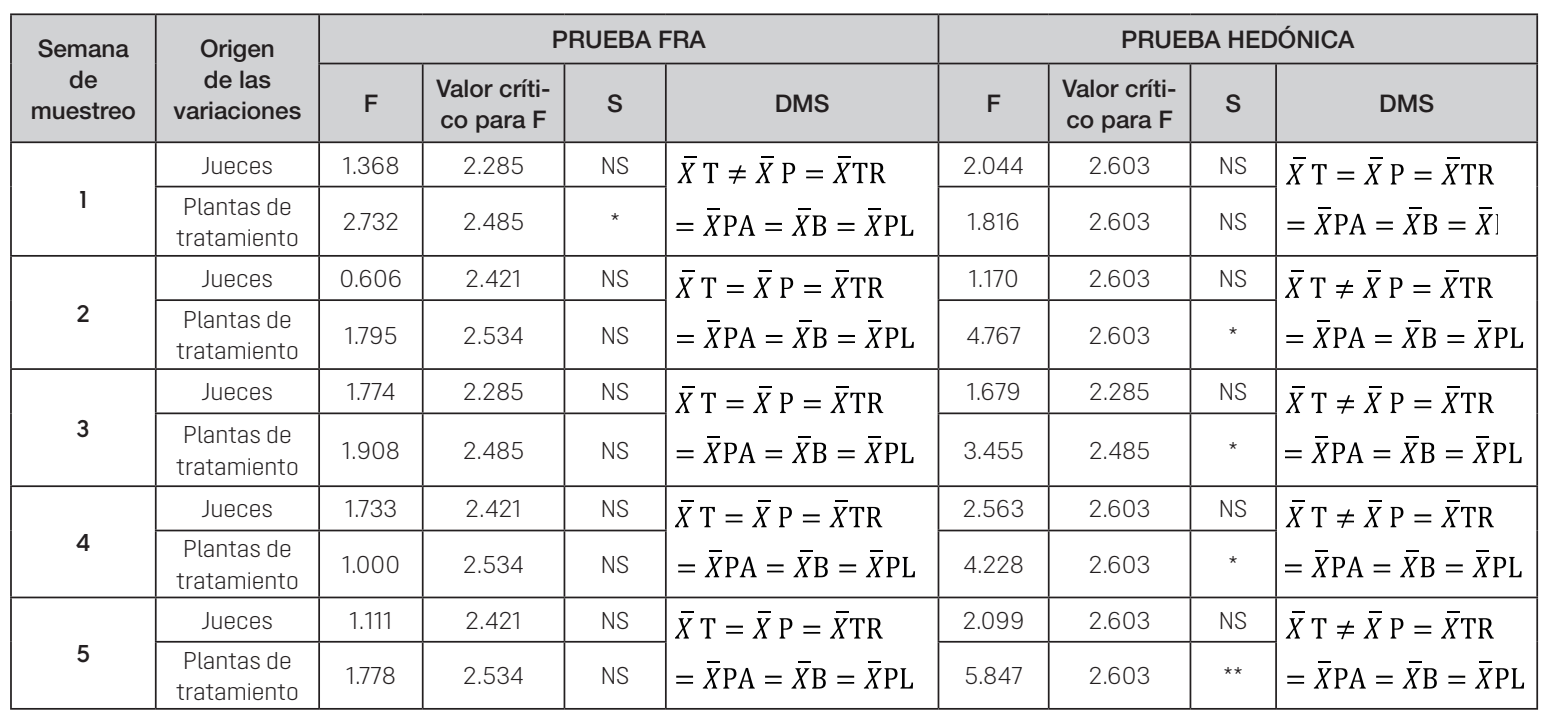

Donde B: PT1, T: PT2, P: PT3, TR: PT4, PL: PT5 y PA: PT6. S: significancia. Jueces: catadores sensoriales entrenados. 
Para correlacionar el grado de aceptación del sabor con el contenido de minerales en el agua potable, se aplicó el análisis de componentes principales (ACP), cuyo propósito fue obtener un número reducido de combinaciones lineales de las 12 variables que expliquen la mayor variabilidad en los datos. En este caso, se extrajeron tres componentes que tuvieron valores propios mayores o iguales a 1.0 y que explican el $84.003 \%$ de la variabilidad en los datos originales.

La figura 2 es una gráfica de proyección de la prueba FRA que sobrepone las puntuaciones (cada planta de tratamiento corresponde a un color), donde las cargas o vectores están marcadas con rojo (minerales y grado de aceptación del sabor), que permite evaluar la estructura de los datos. Se observa que las muestras de PT1 (marcado con azul) y PT6 (marcado con rojo), tienen apreciaciones sensoriales similares y se acercan medianamente a un sabor aceptable (punto de origen de las líneas rojas). Las muestras de PT4 (marcado con morado) y PT3 (marcado con gris) presentan mayor agrupación y son las más cercanas al punto de aceptación del sabor, mientras que las muestras de PT2 (marcado con amarillo), tienen altas concentraciones de $\mathrm{K}^{+}, \mathrm{Ca}^{2+}, \mathrm{Na}^{+}, \mathrm{Mg}^{2+}, \mathrm{CaCO}_{3}, \mathrm{HCO}_{3^{\prime}} \mathrm{Cl}^{-}$y NO${ }_{3}^{-}$y se encuentra más lejanas. Estos resultados son similares a los obtenidos en el estudio realizado en 2013 por Platikanov et al., donde la percepción del sabor se relacionó directamente con la presencia de los iones $\mathrm{HCO}_{3}{ }^{-}, \mathrm{SO}_{4}^{2-}, \mathrm{Ca}^{2+}, \mathrm{Na}^{+}, \mathrm{K}^{+}$ y $\mathrm{Cl}$; igualmente, el cloro residual no produjo ningún efecto en el sabor.

Figura 2. Gráfica de doble proyección de las variables minerales y grado de aceptación del sabor de las muestras de agua potable

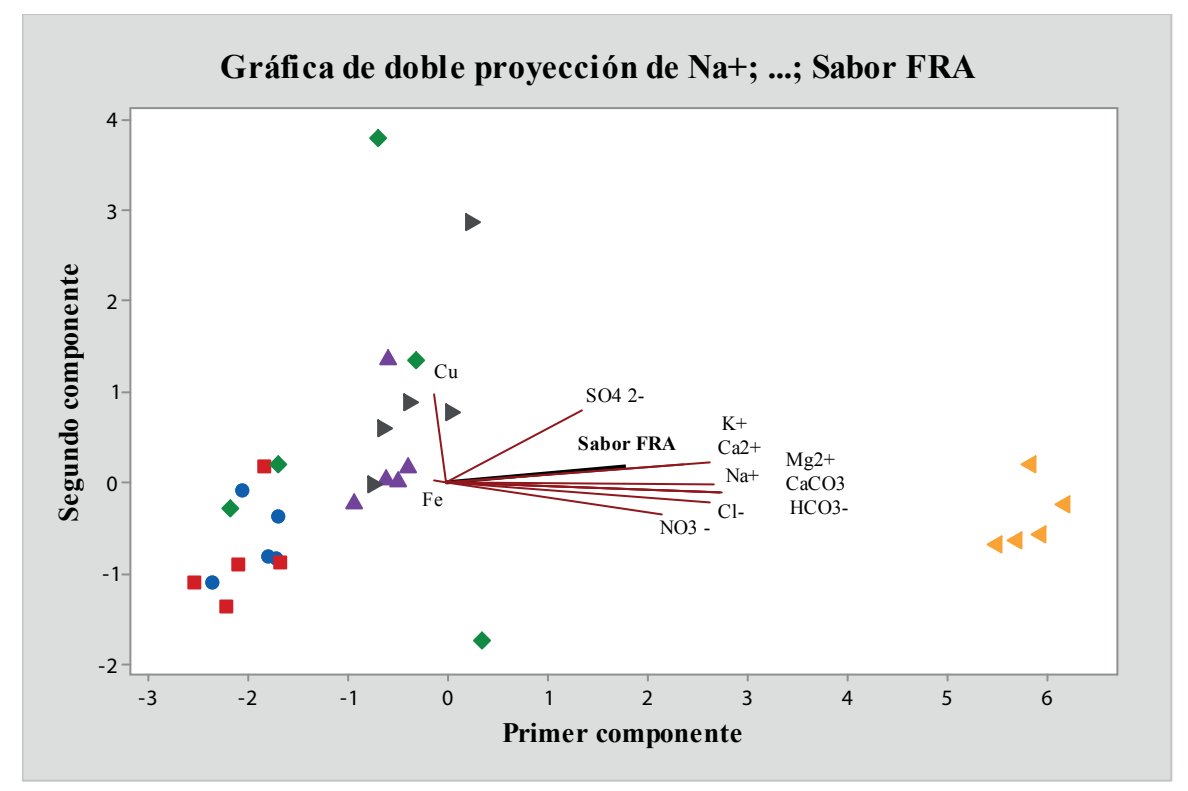

De igual manera, se utilizó una gráfica de doble proyección (figura 3) para evaluar el grado de satisfacción obtenido en la prueba hedónica, para lo que se extrajo tres componentes principales que tuvieron valores propios mayores o iguales a 1.0; los que explican el $86.607 \%$ de la variabilidad en los datos originales. Al igual que en la prueba FRA, las muestras de PT2 (marcado con amarillo), se encuentran más alejadas del punto de satisfacción del sabor. PT1 (marcado con azul) y PT6 (marcado con rojo) presentan resultados cercanos en cuanto al grado de satisfacción del sabor; mientras que PT4 (marcado con morado) y PT3 (marcado con plomo), se acercan más al punto de aceptación del sabor, al igual que PT5 (marcado con verde), a pesar de su dispersión. 
Figura 3. Gráfica de doble proyección de las variables minerales y grado de satisfacción del sabor

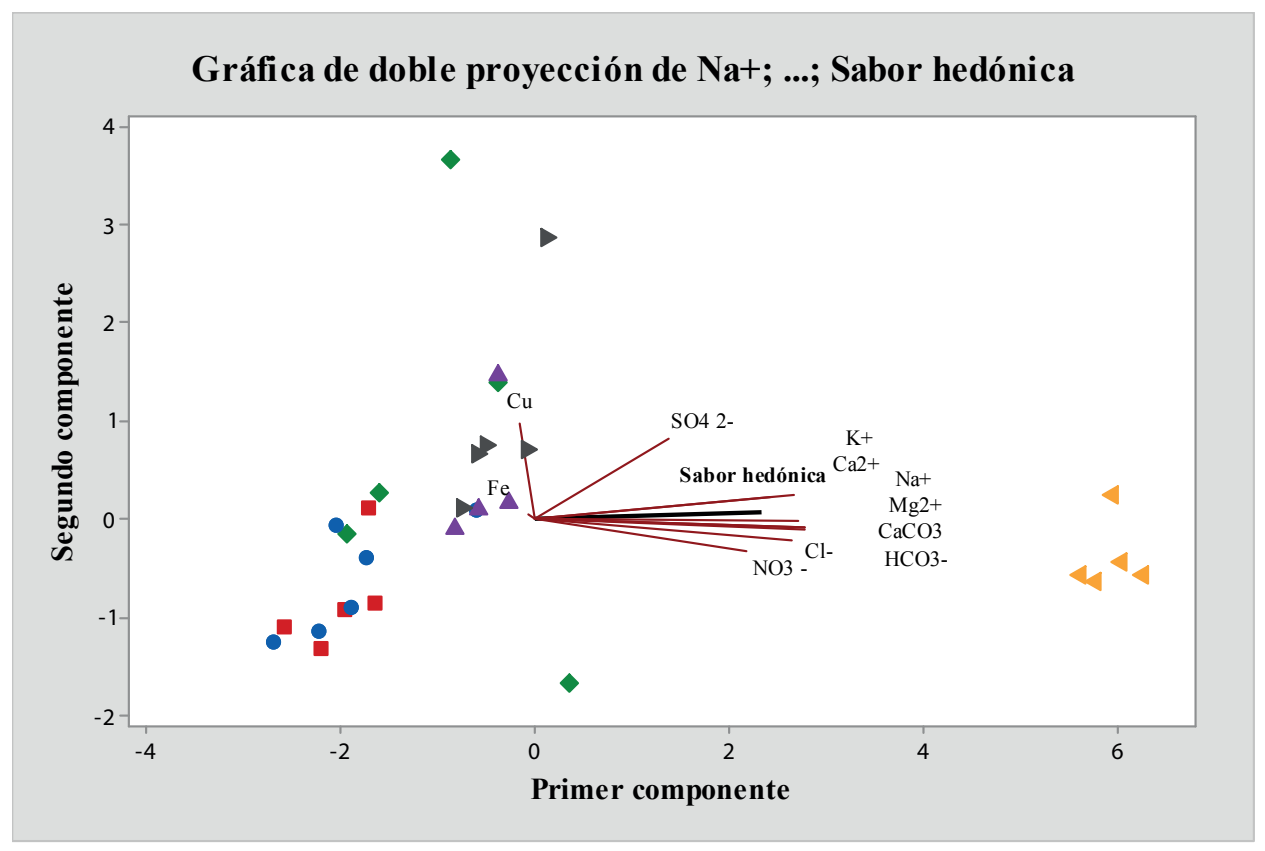

Las dos figuras muestran la relación existente entre la percepción del sabor por parte de los jueces y la concentración de minerales. Al igual que en el estudio de Harmon en 2018 se concluye que las concentraciones bajas de $\mathrm{Ca}^{2+}$ y $\mathrm{HCO}_{3}$-en todas las muestras, a excepción de PT2, se relacionan con mayor preferencia sensorial.

De manera similar a lo descrito en 2017 por Virgerhoeds et al., los minerales presentes en el agua cruda como: $\mathrm{Ca}^{2+}, \mathrm{Mg}^{2+}, \mathrm{CaCO}_{3}, \mathrm{HCO}_{3}-\mathrm{SO}_{4}{ }^{2-}, \mathrm{Cl}^{-}$aportan significativamente a la variación del sabor en el agua potable, ya que permanecen en cantidades detectables luego del tratamiento, además, en este caso las cantidades de $\mathrm{Fe}^{3+} \mathrm{y} \mathrm{Cu}^{2+}$ disminuyen a valores no cuantificables por el método utilizado y tampoco se percibió un sabor metálico en ninguna de las muestras.

Al contrastar con lo expuesto en el estudio de López et al., en 2017, donde los jueces indicaron que el agua tiene sabor salado debido a la presencia de $\mathrm{SO}_{4}{ }^{2-}$ en este caso a pesar de que el valor de $\mathrm{SO}_{4}^{2-}$ de las muestras de PT3 es mayor que las otras plantas, no se perciben como saladas, debido a que contienen menos de $80 \mathrm{mg} / \mathrm{l}$ de este mineral. Las concentraciones de $\mathrm{Na}^{+}$de PT2 se relacionaron con un sabor ligeramente salado descrito por los jueces, similar a lo ocurrido en el artículo de revisión sobre los olores y sabores del agua para beber (Dietrich y Burlingame, 2020).

Según los resultados de las dos pruebas sensoriales, el agua potable de PT2 tiene una ligera diferencia de aceptación y satisfacción en cuanto a su sabor, sin embargo, contiene mayor concentración de minerales y por tal razón, otorga mayor valor nutricional. El aporte diario al consumir dos litros de agua de PT2 sería en promedio de $2.8 \%$ de sodio, $0.2 \%$ de potasio, $2.2 \%$ de calcio, $11.9 \%$ de magnesio y $1.8 \%$ de cloruro del valor diario recomendado (VDR) según (INEN 1334-2, 2008), resultados que serían menores a los indicados en los estudios de Perry et al. en 2013 y de Azoulay et al. en 2001, quienes demostraron que al consumir dos litros de agua de grifo en Estados Unidos, el agua aporta de 8 a $16 \%$ del valor diario de $\mathrm{Ca}^{2+}$ y entre 6 - $31 \%$ de $\mathrm{Mg}^{2+}$. 


\section{Conclusiones y recomendaciones}

Se demostró que la concentración de minerales influye en el sabor del agua potable de las plantas de tratamiento de la Epmaps y que los minerales que más influyen en el sabor son $\mathrm{Ca}^{2+}, \mathrm{Mg}^{2+}$, $\mathrm{Na}^{+}, \mathrm{K}^{+}, \mathrm{HCO}_{3}^{-}, \mathrm{CaCO}_{3}$ y $\mathrm{Cl}^{-}$.

La determinación de parámetros generales permitió relacionar de manera directamente proporcional a la conductividad y los sólidos totales disueltos con la cantidad de minerales, y por tanto con el sabor del agua.

Al realizar la evaluación sensorial mediante dos tipos de pruebas, la hedónica y la prueba FRA, se concluye que la prueba hedónica de nueve puntos permite obtener resultados con diferencias significativas en relación con la concentración de minerales y el sabor percibido, mientras que la prueba FRA de cinco puntos no logra demostrar el objetivo de este estudio, por lo que es recomendable utilizar la prueba hedónica de nueve puntos.

El menor grado de satisfacción se obtuvo en las muestras de la planta de tratamiento 2 , debido a sus mayores concentraciones de $\mathrm{Ca}^{2+}, \mathrm{Mg}^{2+}, \mathrm{Na}^{+}, \mathrm{K}^{+}, \mathrm{HCO}_{3}{ }^{-}, \mathrm{CaCO}_{3}$ y $\mathrm{Cl}$, que a criterio del grupo de catadores se percibió con un sabor "ligeramente salado", lo que permitió comprobar la hipótesis de trabajo propuesta inicialmente.

Al comparar los resultados de este estudio con la norma NTE INEN 1108:2020, se concluye que todas las muestras de agua potable analizadas cumplieron con los requisitos de sabor, color, turbiedad, cloro libre residual, $\mathrm{Cu}^{2+}$ y $\mathrm{NO}_{3}^{-}$, que son parámetros indicadores de calidad establecidos en dicha norma.

Se recomienda realizar estudios similares en el resto del país para conocer las características del agua cruda y tratada de varias ciudades y regiones del Ecuador, lo que permitirá determinar la influencia de parámetros como la altitud, tipo de fuente de agua, localización, en el sabor del agua potable y en los productos elaborados con la misma.

\section{Referencias}

Azoulay A., G. P. (2001). Comparison of the Mineral Content of Tap Water and Borrled Waters. Journal of the General Internal Medicine 16, 168-175.

Burlingame G., D. R. (2017). Humans as Sensors to Evaluate Drinking Water Taste and Odor: A Review. American Water Works Association (109), 13-22.

Dietrich y Burlingame. (2014, December 17). Critical Review and Rethinking of Usepa Secondary Standards for Maintaining Organoleptic Quality of Drinking Water. Environmental Science and Technology 49(2), 708-720. doi: 10.1021/es504403t

Dietrich y Burlingame. (2020, October 17). A Review: The Challenge, Consensus, and Confusion of Describing Odors and Tastes in Drinking Water. Vol. 713. Science of the Total Environment 713, doi: 10.1016/j.scitotenv.2019.135061

Dinelli E., L. A. (2012, January). Comparative Study Between Bottled Mineral and Tap Water in Italy. Journal of Geochemical Exploration 112, 368-389. doi: 10.1016/j.gexplo.2011.11.002

Franks, M. L. (2019). The Influence of Water Composition on Flavor and Nutrient Extraction in Green and Black Tea. Nutrients 11(1): 80.

Harmon D., G. M. (2018, October 28). Preference for Tap, Bottled, and Recycled Water: Relations to PTC Taste Sensitivity and Personality. Appetite(3672), 119-128. doi:10.1016/j.appet.2017.10.040.

INEC, I. N. (2013). Accesibilidad y uso del agua en los hogares ecuatorianos, por regiones naturales. Quito: Instituto Nacional de Estadística y Censos.

INEN 1108, N. (2020). Agua para consumo humano (6. ${ }^{\circ}$ rev.) Requisitos. Quito-Ecuador: INEN. 
INEN 1334-2. (2008). Rotulado de productos alimenticos para consumo humano. Parte 2. Rotulado nutricional. Requisitos. Quito: INEN.

INEN ISO 9963-1. (2014). Calidad del agua, determinación de la alcalinidad. Quito.

López et al. (2017). Effects of Sulfate and Nitrate on the Taste of Water: A Study with a Trained Panel. Research and Technology AQUA, 598-605.

Nagpure et al. (2017). Physico-chemical Analysis of water Samples and Effect of its Quality on Human Health. Life Science Informatics Publication.

NTE INEN 974. (2016). Agua potable. Determinación de la dureza total por titulación con EDTA (1. rev.). Quito: INEN.

OMS, O. M. (2006). Aspectos químicos (3. ed.), Vol. 1. Suiza: Primer apéndice.

Perry, K. Y. (2013, August). The Mineral Content of Tap Water in United States Households. Journal of Food Composition and Analysis 31(1), 46-50. doi: 10.1016/j.jfca.2013.03.004

Platikanov S., G. V. (2013, February 1). Influence of Minerals on the Taste of Bottled and tap Water: A Chemmetric Approach. Water Research 47, 47(2), 693-704. doi: 10.1016/j.watres.2012.10.040

Rahman I., B. S. (2017). Quality Assessment of the Non-Carbonated Bottled Drinking Water Marketed in Bangladesh and Comparison with Tap Water. Food Control, 1149-1158. doi: 10.1016/j.foodcont.2016.10.032

Salguero, L. G.-G.-L.-G. (2013). Training of Panellists for the Sensory Control of Bottled Natural Mineral Water in Connection with Water Chemical Properties. Food Chemistry 141(1), 625 -636. doi: 10.1016/j.foodchem.2013.02.093

Sancho, J. E. (2002). Pruebas de aceptación o hedónicas. Introducción al análisis sensorial de los alimentos (pp. 142-145). Barcelona, España: Alfaomega.

Standard Methods 2120-B. (2012). Standard Methods for the examination of Water and Wastewater. Washington: American Public Health Association.

Standard Methods 2130-B. (2012). Standard Methods for the examination of Water and Wastewater. Washington: American Public Health Association.

Standard Methods 2160-C. (2012). Standard Methods for the examination of Water and Wastewater (22 ed.). Washington: American Public Health Association.

Standard Methods 2510-B. (2012). Standard Methods for the examination of Water and Wastewater. Washington: American Public Health Association.

Standard Methods 3111-B. (2012). Standard Methods for the examination of Water and Wastewater. Washington: American Public Health Association.

Standard Methods 3500-Ca-B. (2012). Standard Methods for the examination of Water and Wastewater. Washington: American Public Health Association.

Standard Methods 3500-Mg-B. (2012). Standard Methods for the examination of Water and Wastewater. Washington: American Public Health Association.

Standard Methods 4500-Cl-B. (2012). Standard Methods for the examination of Water and Wastewater. Washington: American Public Health Association.

Standard Methods 4500-Cl-G. (2012). Standard Methods for the examination of Water and Wastewater. Washington: American Public Health Association.

Standard Methods 4500-H+-B. (2012). Standard Methods for the examination of Water and Wastewater. Washington: American Public Health Association.

Standard Methods 4500-NO3-B. (2012). Standard Methods for the examination of Water and Wastewater. Washington: American Public Health Association.

Standard Methods 4500-SO4-E. (2012). Standard Methods for the examination of Water and Wastewater. Washington: American Public Health Association.

USEPA. (2020). Secondary Drinking Water Standards: Guidance for Nuisance Chemicals. Retrieved on 042020, from https://bit.ly/2Z74F8Q 
Villarroel, L. A. (2003). Aplicación del analisis de componentes principales en el desarrollo de productos. Cochabamba - Bolivia: UMSS.

Vingerhoeds, M. (2017). Sensory Quality of Drinking Water Produced by Reverse Osmosis Membrane Filtration Followed by Remineralisation. Water Research, 42-51.

Wang A., D. S. (2016, December). Effect of Iron on Taste Perception and Emotional Response of Sweetened Beverage under Different Water Conditions. Food Quality and Preference 54, 58-66. doi: 10.1016/j.foodqual.2016.06.016 